\title{
Design for GSM-based Motor Remote Monitoring Device
}

\author{
Yu-qiao MENG \\ Jilin Teachers Institute of Engineering and Technology, Triumph Road No. \\ 3050, changchun, 130052. China
}

942970277@qq.com

Keywords: GSM, Motor, Remote Monitoring, Detector

\begin{abstract}
This paper is focused on a GSM-based motor remote-monitoring device designed on the basis of convenient and safe motor management to help improve motor safety and prolong service life. It is able to monitor operation of multiple motors simultaneously in an effective manner and thus control them remotely. The overall structural diagram of design and function block of detector are introduced in this paper. Featured by easy assembly, simple wiring, low cost and good expandability, this system can have better application value.
\end{abstract}

\section{Background}

Motors are used frequently in national industry, such as household appliances, automotive electronics, spinning, avionics and machinery industry, as well as people's lives. There can be huge waste of manpower if motors which are always used for long time at severe environment are monitored manually. Without the ability of self protection, the motors, if suffering from a fault due to unstable power grid and aging, may have adverse influences on normal production and even endanger workers' personal safety. Although some motor monitoring devices can only help operators monitor motors in production line, it is unable to shut down motor in case of an accident; the motors are still likely to be damaged if operated for long time, not to mention the monitoring to multiple motors. Basically, the multiple motors are simultaneously monitored by integrating industrial personal computer (IPC) or in a way combined with PC and upper computer software. It has short development period, but has such disadvantages as large occupation of computer resources, complex wiring, high cost and bad portability. That's why it is becoming increasingly important to make simultaneous monitoring, protection and management to multiple motors in an economical, efficient and easy way.

\section{Characteristics of GSM Network}

The appearance of the concept Internet of Things (IOT) brings a spring for developing remote monitoring technology. As a branch of remote monitoring technology, remote motor monitoring has wide and profound application foreground. Moreover, the remote monitoring technology is constantly innovated by the rapid development of wireless network communication, in which, GSM is the leading $2 \mathrm{G}$ standard worldwide. GSM is a mature and widely-used system based on multi-address technique-based mobile communication. The GSM short message service (SMS) system is characterized by wide coverage, high reliability, short demonstration time and no restrain from communication distance. In case there's network fault and user's data cannot be sent to opposite mobile terminal, SMSC can help protect user's data by firstly storing it and then transiting 
SMS if network is recovered. Therefore, it can offer high-quality communication at low cost of service and terminal.

\section{Design for GSM-based Motor Remote Monitoring Device}

GSM-based Motor Remote Monitoring Device (hereinafter referred to as "this Device") is composed of upper computer and at least one site terminal detector. The terminal detector and upper computer are connected via wireless network circuit. The quantity of site terminal detector 2 can be changed according to quantity of to-be-monitored motors. The schematic diagram for structure of this Device is as shown in Fig. 1, in which, 1- upper computer, 2-site terminal detector.

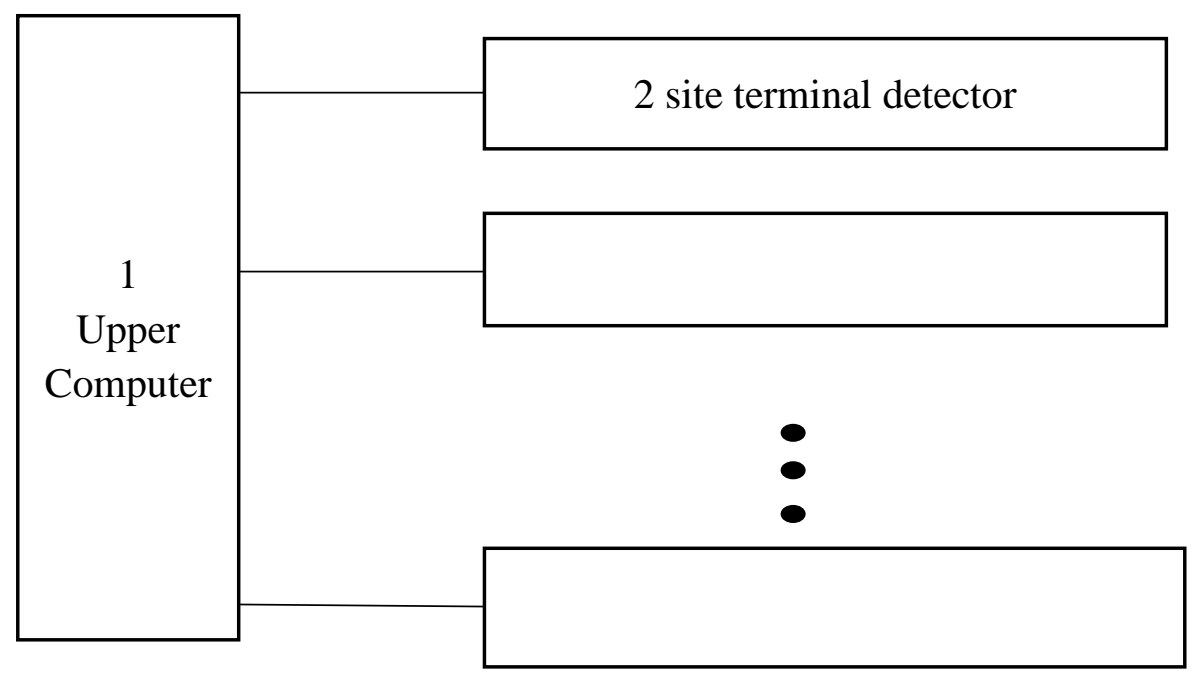

Fig.1 Schematic diagram of monitoring device structure

Site terminal launcher is composed of single-chip microcomputer (SCM) module, GPS positioning module, power module, key module, rotation speed display unit, timing power-off module, sensor module, alarm module, detection sampling module, GSM module and user's mobile phone. The structure of site terminal launcher is shown in Fig. 2. The power module is electrically connected with GPS positioning module, key module, rotation speed display unit, timing power-off module, sensor module, detection sampling module and GSM module. SCM module is electrically connected with the user's mobile phone through GSM module. The GPS positioning module, key module, rotation speed display unit, timing power-off module, sensor module, alarm module and detection sampling module have electric connection to the sensor module. 


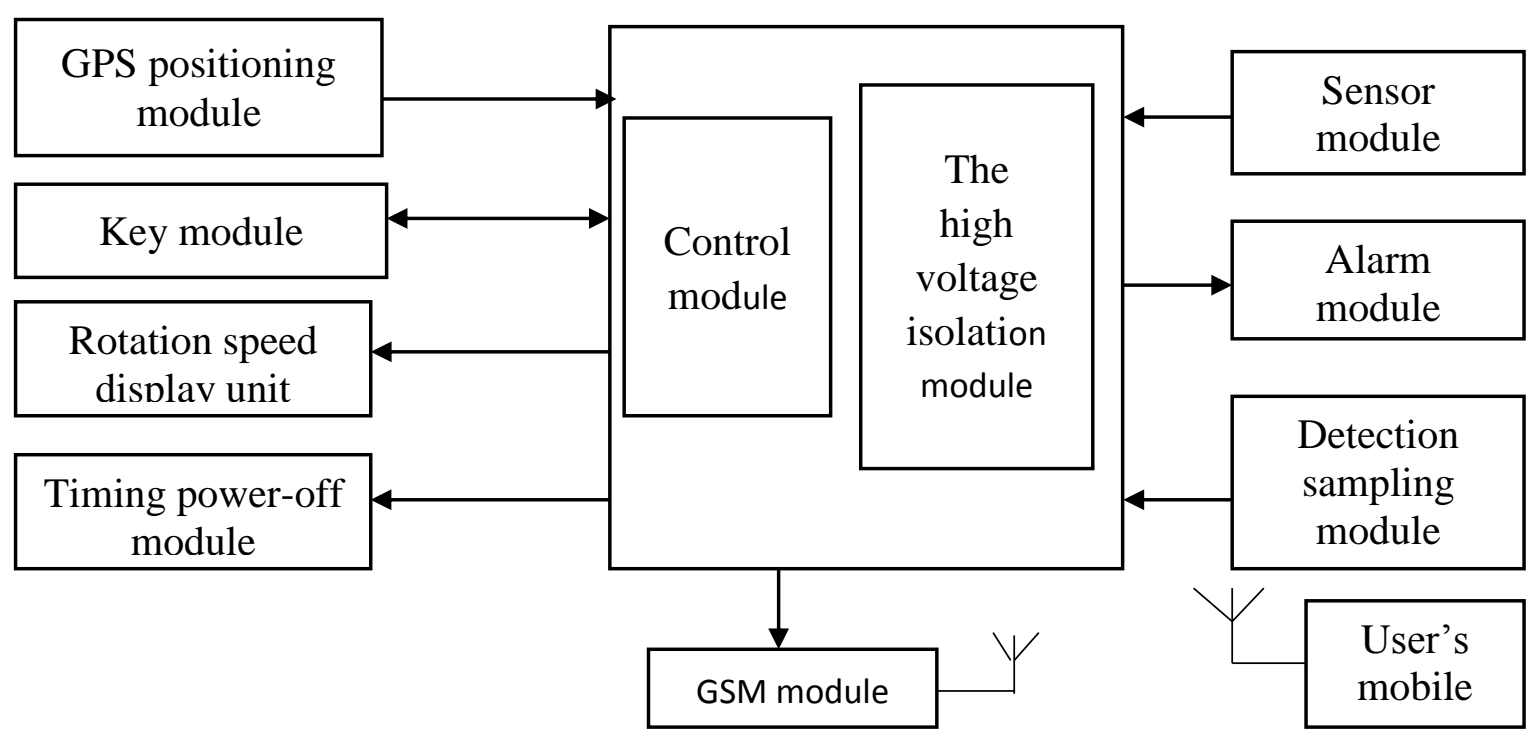

Fig.2 Terminal lauuncher structure diagram

The users can find accurate position of motor using GPS positioning module, regulate motor rotation speed by key module, observe motor rotation speed by rotation speed display unit, test motor operation parameters in virtue of detection sampling module and send the test result to upper computer 1 and user's mobile phone through SCM module. The SCM module is composed of control module and high voltage isolation module. The high voltage isolation module can avoid damaging control module and prolong its service life. The sensor module and SCM module are connected with signal conversion module. The sensor module includes fiber optic temperature sensor and humidity sensor. The temperature measuring optical fiber of fiber optic temperature sensor is bound on the to-be-tested motor through fastening belt in order to regulate the temperature and humidity of working environment and help users know about the conditions of working environment. The control module is installed with the chip of PIC16F690. The character type LCD is adopted by rotation speed display unit. The chip for key circuit is ZLG7289.

\section{Advantages of GSM-based Motor Remote Monitoring Device}

This Device can test operation data of motor and surrounding parameters and upload such data to upper computer 1 and user's mobile phone. On basis of timing cut-off module and strong current isolation module, this Device can protect the motor and control modules and prevent too long or continuous operation of operation in order to improve safety. The users can regulate motor rotation speed through key module and observe motor rotation speed through rotation speed display unit, making this Device more flexible. The alarm module can help patrol inspectors timely find out the fault position for handling.

In this paper, a GSM-based motor remote monitoring device is designed by integrating the modern communication technique in order to simultaneously remote multiple motors, which can help the professional staffs detect and eliminate the fault at early stage. According to the test, this Device can significantly improve test efficiency, reduce manpower and financial investment in monitoring and test and increase the safety. 


\section{References}

[1] Liu Fangyu, Research of Smart GSM/GPRS Remote Motor Monitoring System, Journal of Liaoning Teachers College: Natural Science Edition, March, 2013

[2] Liu Jingyu, Design and Development of Remote Intelligent Motor Monitoring and Protection Device, East China University of Science and Technology, 2011

[3] Fang Shubin, Design and Research for Smart Monitoring Device of Motor, Hunan University, 2013

[4] Deng Zhenjie, Design of remote monitoring and control system based on GSM, Micro computer information, 2007

[5] Lin Chenghua,Remote control system based on GSM, Electrical automation,2012 